\title{
Modern Lattice Boltzmann Methods for Multiphase Micro-Flows
}

\author{
G. Falcucci ${ }^{1,2}$, S. Ubertini ${ }^{1}$, D. Chiappini ${ }^{2}$ and S. Succi ${ }^{3, *}$ \\ 1 Dept. of Technologies, \\ University of Naples "Parthenope", \\ Centro Direzionale, Isola C4, Naples, Italy \\ 2 Dept. of Mechanical Engineering, \\ University of Rome "Tor Vergata", \\ V.le Politecnico 1, Rome, Italy \\ 3 Istituto Sistemi Complessi - CNR, \\ Via dei Taurini 19, 00185, Rome, Italy \\ * corresponding author
}

(Dated: August 5, 2009)

\begin{abstract}
During the last decade, the Lattice Boltzmann (LB) method has captured an increasing attention as an efficient tool for the numerical simulation of complex fluids, particularly multi-phase and multi-component flows. In this paper, we revisit the basic features of two modern variants of lattice Boltzmann models for non-ideal fluids, which offer promising perspectives for the numerical simulation of complex micro-flows.
\end{abstract}

\section{INTRODUCTION}

In the last decade, Lattice Boltzmann models for non-ideal fluids have captured a growing interest for the simulation of complex multiphase/multicomponent flows. In particular, the Shan-Chen pseudo-potential $(P P)$ method [1], and the free-energy $(F E)$ approach $[2,3]$, have made the object of many investigations and applications. Likewise their single-phase counterpart, these models are finding increasing applications for problems at the microscale, mostly on account of their ability to flexibly incorporate microscopic physics beyond the realm of macroscopic fluid dynamics, while still preserving their computational efficiency $[4,5]$. Nevertheless, some recognized weaknesses of such schemes are possibly accrued by moving from the macro to the microscale. For instance, the $P P$ method has no easy access to small surface tensions and consequently it cannot generate (metastable) multidroplet configurations, which are crucial to many microfluidic applications. In addition, it is affected by spurious currents near phase interfaces which may obscure the physical behaviour of the microfluid, especially near solid surfaces, where spurious currents become comparable to the fluid speed [6]. In this paper, we discuss some aspects of two modern variants of LB schemes for multiphase fluids, namely the multi-range pseudopotential method, and the free-energyfinite-difference scheme, which considerably mitigate the aforementioned limitations. In particular, the so-called multi-range Shan-Chen model, by including the competing effect of short-range attraction and mid-range repulsion, permits to achieve significant reduction of the surface tension, thereby opening the way to the simulation of multidroplet fluid configurations with tunable droplet size. On the other hand, finite-difference extensions of the $F E$ method, 
have proven capable to significantly reduce the intensity of spurious currents by a higher-order treatment of capillary forces. Both methods have significant potential ahead for microfluidic applications. An optimal combination of the two might gain the status of the premiere LB model for complex microfluidics.

\section{THE LATTICE BOLTZMANN METHOD}

The Lattice Boltzmann method is based on a minimal discretization in velocity space of the continuum Boltzmann equation in relaxation form, namely:

$$
\begin{aligned}
\frac{\partial f_{\alpha}(\boldsymbol{x}, t)}{\partial t} & +\boldsymbol{c}_{\alpha} \nabla f_{\alpha}(\boldsymbol{x}, t)= \\
& -\frac{1}{\tau}\left[f_{\alpha}(\boldsymbol{x}, t)-f_{\alpha}^{e q}(\boldsymbol{x}, t)\right]
\end{aligned}
$$

where $f_{\alpha}(\boldsymbol{x}, t)$ is the particle distribution function $(D F)$ at the site $\boldsymbol{x}$ and time $t$ moving with speed $c$ along the direction $\boldsymbol{c}_{\alpha}$ (9 for $2 \mathrm{D}$ simulation and 15 or 19 for 3D, [7]) associated with a uniform Cartesian lattice. In eq. (1), $\tau$ is the (single) relaxation time to a local equilibrium $f_{\alpha}^{e q}(\mathbf{x}, t)$, depending on the macroscopic flow variables according to the following expression:

$$
\begin{aligned}
f_{\alpha}^{e q}(\boldsymbol{x}, t) & = \\
w_{\alpha} \rho(\boldsymbol{x}, t) & {\left[\frac{\boldsymbol{c}_{\alpha} \cdot \boldsymbol{u}(\boldsymbol{x}, t)}{c_{s}^{2}}+\right.} \\
+ & \left.\frac{\left[\boldsymbol{c}_{\alpha} \cdot \boldsymbol{u}(\boldsymbol{x}, t)\right]^{2}}{2 c_{s}^{4}}-\frac{[\boldsymbol{u}(\boldsymbol{x}, t) \cdot \boldsymbol{u}(\boldsymbol{x}, t)]}{2 c_{s}^{2}}\right]
\end{aligned}
$$

where $w_{\alpha}$ is a set of weights normalized to unity. Explicit time marching of (1) yields the following discrete form:

$$
\begin{aligned}
\Delta_{\alpha} f_{\alpha}(\boldsymbol{x}, t) & \equiv f_{\alpha}\left(\boldsymbol{x}+\boldsymbol{c}_{\alpha} \Delta t, t+\Delta t\right)-f_{i}(\boldsymbol{x}, t)= \\
& -\frac{\Delta t}{\tau}\left[f_{\alpha}(\boldsymbol{x}, t)-f_{\alpha}^{e q}(\boldsymbol{x}, t)\right]
\end{aligned}
$$

The fluid mass and momentum density are defined as moments of $f_{\alpha},[8]$ :

$$
\begin{gathered}
\rho(\boldsymbol{x}, t)=\sum_{\alpha=0}^{N-1} f_{\alpha}(\boldsymbol{x}, t) \\
\rho \boldsymbol{u}(\boldsymbol{x}, t)=\sum_{\alpha=0}^{N-1} \boldsymbol{c}_{\alpha} f_{\alpha}(\boldsymbol{x}, t)
\end{gathered}
$$

where $N$ denotes the number of discrete velocities. In the limit of weak departures from local equilibrium (i.e. small Knudsen numbers) and near the incompressible limit (small Mach numbers), it can be shown [[8]] through the Chapman-Enskog expansion that the above formulation recovers the dynamic behaviour of an ideal fluid with pressure $P=c_{s}^{2} \rho$ and kinematic viscosity $\nu=c_{s}^{2}(\tau-\Delta t / 2)$, where $c_{s}^{2}$ is the lattice sound speed.

The LB method allows to account for the interactions between different phases in non-ideal fluids, by means of a generalized force term in eq. (1):

$$
\frac{\vec{F}}{m} \cdot \frac{\partial f}{\partial \boldsymbol{v}}
$$

in which the generalized force $\vec{F}$, accounts for both external fields (such as gravity or electromagnetic fields) and self-consistent forces associated with intermolecular interactions or any other effective interaction one may wish to include. The corresponding generalized LB equation reads as follows:

$$
\Delta_{\alpha} f_{\alpha}=-\frac{\Delta t}{\tau}\left(f_{\alpha}-f_{\alpha}^{e q}\right)+S_{\alpha} \Delta t
$$


where $S_{\alpha}$ is a source term that can be formally interpreted as the discrete representation of (6). Operationally, $S_{\alpha}$ can be a source of mass, momentum, energy, or any other macroscopic observable in the form:

$$
S(\boldsymbol{x}, t)=\sum_{\alpha} s\left(\boldsymbol{c}_{\alpha}, \boldsymbol{x}\right) f_{\alpha}(\boldsymbol{x}, t)
$$

where $s\left(\boldsymbol{c}_{\alpha}, \boldsymbol{x}\right)$ is a generic phase-space dependent microscopic quantity. Different formulations/implementations of the term $S_{\alpha}$, give rise to a series of distinct multiphase approaches in the Lattice Boltzmann class of methods.

III. THE

PSEUDO-POTENTIAL PROACH

The Pseudo-Potential Approach starts with the standard lattice Boltzmann (LB) equation (1) with pseudo-potentials [1,9]:

$$
\begin{aligned}
& f_{\alpha}\left(\boldsymbol{x}+\boldsymbol{c}_{\alpha} \Delta t, t+\Delta t\right)-f_{i}(\boldsymbol{x}, t)= \\
& -\frac{\Delta t}{\tau}\left[f_{\alpha}(\boldsymbol{x}, t)-f_{\alpha}^{e q}(\boldsymbol{x}, t)\right]+F_{\alpha}(\boldsymbol{x}, t) \Delta t
\end{aligned}
$$

The term $F_{\alpha}$ is a pseudo-force term, whose expression is given below:

$$
\boldsymbol{F}(\boldsymbol{x}, t)=-G_{0} \psi(\boldsymbol{x}, t) \sum_{\alpha=0}^{N-1} \psi\left(\boldsymbol{x}+\boldsymbol{c}_{\alpha} \Delta t, t\right) \boldsymbol{c}_{\alpha} w_{\alpha}
$$

where $N=9$ is the number of discrete speeds in two dimensions and $\psi(\boldsymbol{x}, t)$ is a local functional of the fluid density:

$$
\psi(\boldsymbol{x}, t)=\rho_{0}\left[1-e^{\left(-\frac{\rho(\boldsymbol{x}, t)}{\rho_{0}}\right)}\right]
$$

In this application the reference density $\rho_{0}$ is set to $\rho_{0}=1$ and $G_{0}$ is the basic parameter which rules the inter-particle interaction. In this model, $G_{0}$ is the only free parameter fixing both liquid to vapor density ratio and surface tension.
The possibility of modifying these parameters is limited to a short range of values near the critical ones ensuring phase separation.

Starting from Eq. (10) the component of the interaction potential along each direction can be evaluated. This force is used to shift the velocities, prior to evaluating the equilibrium distribution functions, according to:

$$
\boldsymbol{u}^{\prime}(\boldsymbol{x}, t)=\boldsymbol{u}(\boldsymbol{x}, t)+\frac{\boldsymbol{F}(\boldsymbol{x}, t) \tau}{\rho(\boldsymbol{x}, t)}
$$

The equation of state of the system reflects the force contribution through a non-ideal term on top of the ideal pressure $c_{s}^{2} \rho$ :

$$
P=\rho c_{s}^{2}+\frac{c_{s}^{2} G_{0}}{2} \psi^{2}
$$

As already noted, the Shan-Chen model does not allow a separate tuning of the equation of state and the surface tension, since both are controlled by the same parameter $G_{0}$. This limitation has been recently lifted, by allowing the non-ideal interaction to extend to the second Brillouin zone ("belt", for simplicity) [10-12].

Specifically, one writes the non-ideal force as the sum of two separate components $\vec{F}(\boldsymbol{x}, t)=$ $\vec{F}_{1}(\boldsymbol{x}, t)+\vec{F}_{2}(\boldsymbol{x}, t)$, defined as follows:

$$
\begin{aligned}
\boldsymbol{F}_{1}(\boldsymbol{x}, t) & =G_{1} \psi(\boldsymbol{x}, t) \sum_{\alpha=0}^{b_{1}} w_{i} \psi\left(\boldsymbol{x}_{1 \alpha}, t\right) \boldsymbol{c}_{1 \alpha} \\
\boldsymbol{F}_{2}(\boldsymbol{x}, t) & =G_{2} \psi(\boldsymbol{x}, t) \sum_{\alpha=0}^{b_{1}} p_{1 \alpha} \psi\left(\boldsymbol{x}_{1 \alpha}, t\right) \boldsymbol{c}_{1 \alpha}+ \\
& +G_{2} \psi(\boldsymbol{x}, t) \sum_{\alpha=0}^{b_{2}} p_{2 \alpha} \psi\left(\boldsymbol{x}_{2 \alpha}, t\right) \boldsymbol{c}_{2 \alpha}(1
\end{aligned}
$$

In the above $\boldsymbol{x}_{k \alpha} \equiv \boldsymbol{x}+\boldsymbol{c}_{k \alpha} \Delta t$, where the discrete speeds with index 1 run over the eight nearest neighbors composing the first belt $\left(b_{1}=\right.$ 8, velocity magnitude 1 and 2), while index 2 refers to the second belt, constituted of 16 nextto nearest neighbors, with magnitudes 4, 5, 8 (see Fig. III). 


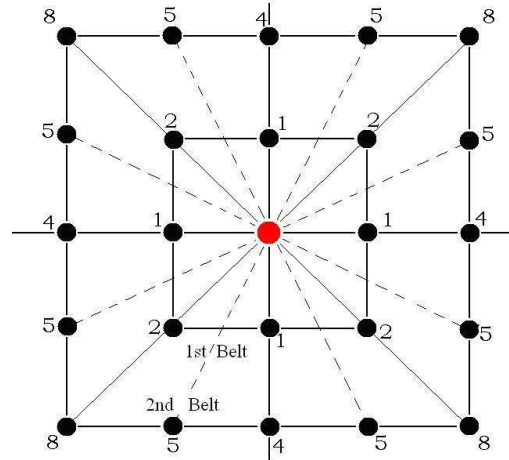

FIG. 1: Two-belt lattice for force evaluation. Each node is labelled by the corresponding energy $\left|c_{\alpha j}\right|^{2}$. Belt 1 contains eight speeds and two energy levels $(1,2)$. Belt 2 contains sixteen speeds, distributed over three energy levels $(4,5,8)$, [[11]].

As is well-known, the standard one-belt, 9speed (including a zero-speed rest particle) lattice provides 4 th-order isotropy, whereas the 2 belts, 24-speed lattice upgrades isotropy to 8 th order, provided the weights are properly chosen. A suitable choice is reported in [11].

We wish emphasize that the 2-belt lattice is used only for the (pseudo)-force evaluation, whereas tha standard lattice Boltzmann dynamics still takes place in the original D2Q9 lattice. This is also the reason why we keep a separate notation for the weights $w_{\alpha}$ used for the lattice Boltzmann populations and the weights $p_{\alpha j}$ used for the force evaluation. The pseudo-potential $\psi(\boldsymbol{x})$ is taken in the form first suggested by Shan and Chen $[1], \psi[\rho]=\sqrt{\rho_{0}}\left(1-e^{-\rho / \rho_{0}}\right)$ where $\rho_{0}$ marks the packing-density, i.e. the density value (criti$\mathrm{cal}$ ) at which non ideal-effects come into play. To be noted that $\boldsymbol{F}_{2}$ acts on both belts, so that, by choosing $G_{1}+G_{2}<0$ and $G_{2}>0$, the present model implements the crucial competition between short-range attraction and mid-range (second belt) repulsion. The former provides the driving force for phase-separation, whereas the latter frustrates this tendency. This competi- tion provides a strong retardation, and sometimes even arrest, of the phase-separation processe, thereby promoting the onset of metastable states, in the form of long-lived multidroplet configurations. With two parameters at hand, $G_{1}$ and $G_{2}$, the present model allows a separate control of the equation of state and surface tension, respectively. In particular, as shown in previous work [13], the non-ideal part of the equation of state depends only on $A_{1}=G_{1}+G_{2}$, whereas surface tension effects are controlled by the combination $G_{1}+\frac{12}{7} G_{2}$. Indeed, Taylor expansion of (14) to second-order delivers the following nonideal equation of state (EOS):

$$
P=\rho c_{s}^{2}+\frac{\left(G_{1}+G_{2}\right)}{2} \psi^{2}(\boldsymbol{x}, t)
$$

Further expansion of eq. (14) to fourth-order provides the following expression for the surface tension:

$$
\left.\gamma=-\frac{\left(G_{1}+\frac{12}{7} G_{2}\right)}{2} c_{s}^{4} \int_{-\infty}^{\infty} \mid \partial_{y} \psi\right)\left.\right|^{2} d y
$$

where the coordinate $y$ runs across the phase interface. The above expressions clearly show that the pressure $P$ and the surface tension $\gamma$ can be changed independently, by properly tuning the interaction parameters $G_{1}$ and $G_{2}$. More specifically, as is well known from the standard Shan-Chen model, phase separation is triggered by choosing $A_{1}<-4$, at a critical density $\rho_{\text {crit }}=\rho_{0} \ln 2$. On the other hand, surface tension can be taken to zero by choosing $G_{2}$ in such a way that $G_{1}+\frac{12}{7} G_{2}=0$. However, since in the vicinity of $\gamma \rightarrow 0$, higher order terms come into play, it proves expedient to introduce a second coupling amplitude:

$$
A_{2}=G_{1}+\lambda G_{2}
$$

where the numerical factor $\lambda$ plays the role of an empirical renormalisation parameter, whose departure from zeroth-order value $\frac{12}{7}$ provides 
a measure of the influence of the higher-order terms. Numerical results indicate that $\lambda \approx 3 / 2$ provides satisfactory agreement [13] with the simulations.

\section{A. Numerical simulations with the Pseudo-Potential method}

In Figure 2 we show the results obtained with the PP approach in the two versions, the standard single-range Shan-Chen and the 2-belts multi-range version. The simulations are performed in periodic boxes of size $512^{2}$, with initial density $\rho_{\text {init }}=\rho_{c r}=\ln 2$, [9], plus a random perturbation of variance $0.01 \rho_{\text {init }}$, to trigger phase-separation. The sequence of figures, from top to bottom, illustrates the effect of decreasing the surface tension (the so-called multidroplet configuration) by letting $A_{2}$ become increasingly less negative and finally change sign (virtually corresponding to negative surface tension). From this sequence of figures, it is apparent that, by lowering the surface tension, an increasing number of droplets of decreasing size is generated (the total mass of the liquid droplet is fixed by the initial conditions and the equation of state).

By further increasing $A_{2}$ towards positive values, droplets of increasingly smaller size are generated, Fig. 3, strongly reminiscent of a quasicrystal or an emulsion, with large scale ordered domains, [13].

The PP approach can also be used to study the wettability of solid surfaces and the behavior of droplets impinging on wet or dry (hydrophobic or hydrophilic) surfaces. To this purpose, the simplest route is to define a separate pseudo-potential for the fluid-wall interactions. The contact angle can then be calibrated as a function of the coupling strength of the fluid- wall interaction [14].

As an example, Figure $4 b$ reports the comparison of the deformation of a droplet impinging on a dry hydrophobic surface (Fig. 4b)), with the experimental measurements performed by Clanet et al. [15]. The Weber number in Fig. $4 b$ is $W e=\rho u^{2} D / \gamma \sim 10$.

The results in Fig. $4 a$ are obtained by considering a droplet with a 20 lattice unit (lu) diameter, with impinging velocities varying from 0.01 to $0.2 l u$, corresponding to a Weber number $W e \sim 0.1-20$, typical of many technical applications (i.e., impinging fuel sprays, coolant systems in gas turbines, painting sprays and inkjets): the good match between the LB simulations and the experimental data is well visible. Such form of fluid-solid coupling proves very useful for microfluidic applications, provided appropriate boundary conditions are used, [17, 18].

Summarizing, the standard Shan-Chen model is simple, elegant and efficient. However, it also suffers of some drawbacks, primarily the fact that the surface tension cannot be tuned independently on the equation of state (hence the density ratio between liquid and gas phases). In addition, it can be readily shown that it cannot access values of the surface tension below $\gamma \sim 0.01$ (in lattice units), mostly on account of the fact that the coupling strength must be kept above a critical threshold, $|G|>4$, to achieve phase-separation. The latter constraint, particularly severe for microfluidic problems, is relaxed to a large extent by the multi-range extensions discussed above.

A further limitation of the SC model is the presence of so-called "spurious currents", i.e non zero-velocity vortex configurations, which owe their existence solely to the lack of high-order isotropy of the lattice (beyond the requirement of Navier-Stokes equations for ideal fluids). To date, there is no evidence 

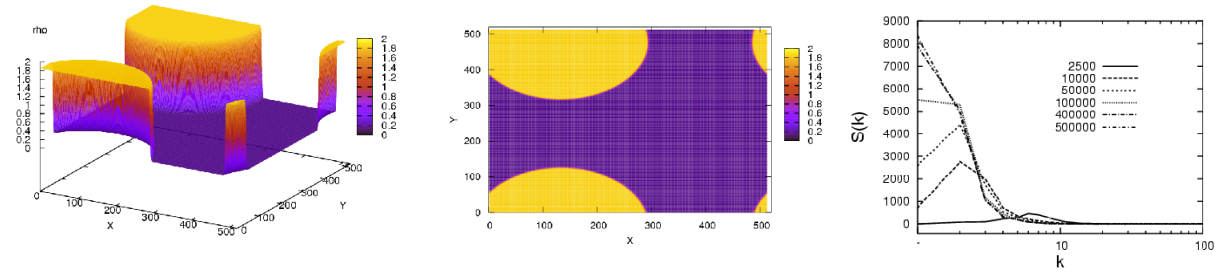

(a)
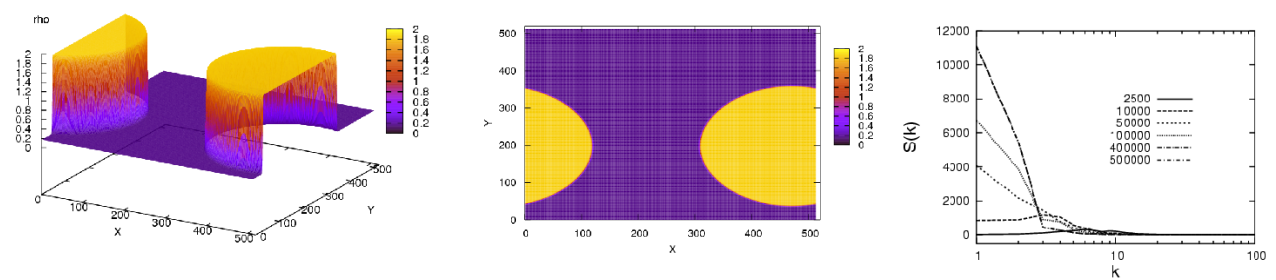

$(b)$
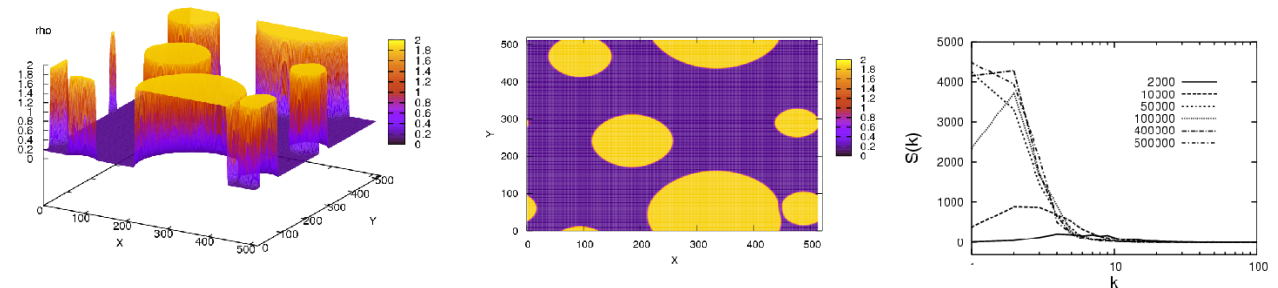

(c)
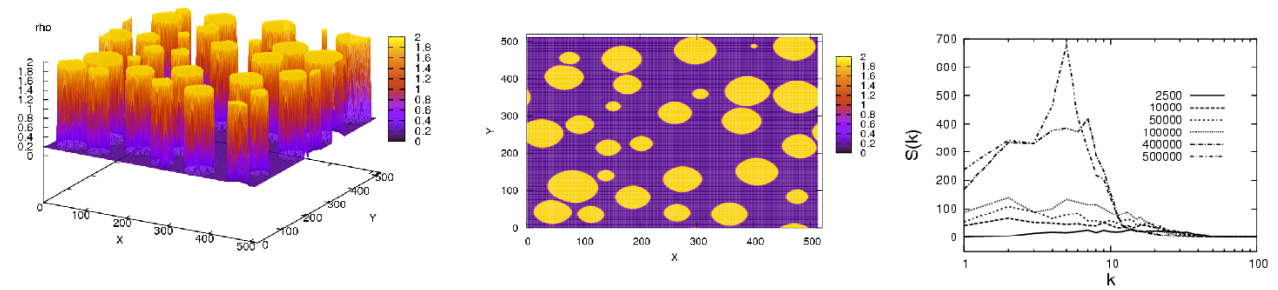

$(d)$

FIG. 2: Spatial distribution of the fluid density. The formation of a large number of droplets with increasing $A_{2}$ is well visible. [a]: Standard Shan-Chen, $A_{1}=-4.9, A_{2}=-4.9 n x=n y=512, t=500000$; [b]: Multi-droplet, $A_{2}=-2.85, n x=n y=512, t=500000$; [c]: Multi-droplet, $A_{2}=-0.8, n x=n y=512$, $t=500000$; [d]: Multi-droplet, $A_{2}=0.15, n x=n y=512, t=500000$. The right panel shows the Fourier spectrum of density fluctuations. Such spectrum, initially a white noise, evolves towards a shape peaked at the (inverse) size of the droplets. These Fourier spectra show that small-scale contribution is significantly

higher when increasing the mid-range repulsion, that is $A_{2}$, indicating the formation of long-lived metastable states in the form of small droplets. In particular in the last picture (d), at the end of the simulation there is a clear peak at $R \sim n x / 2 k \approx 30,[13]$. 

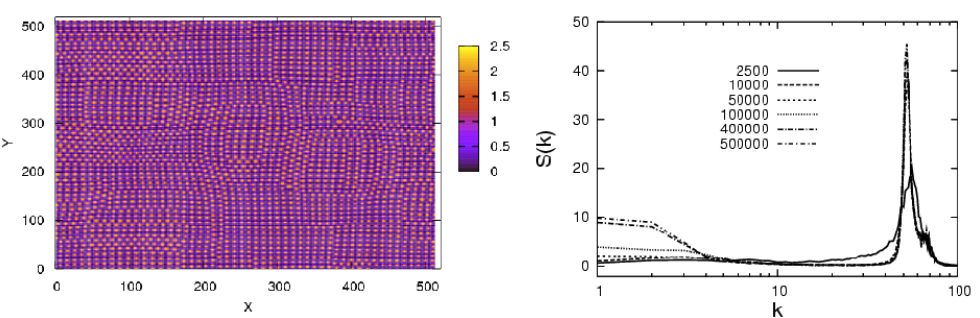

(a)
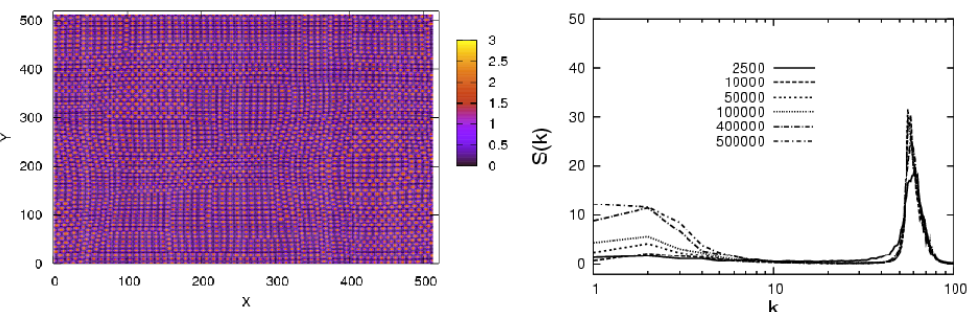

(b)
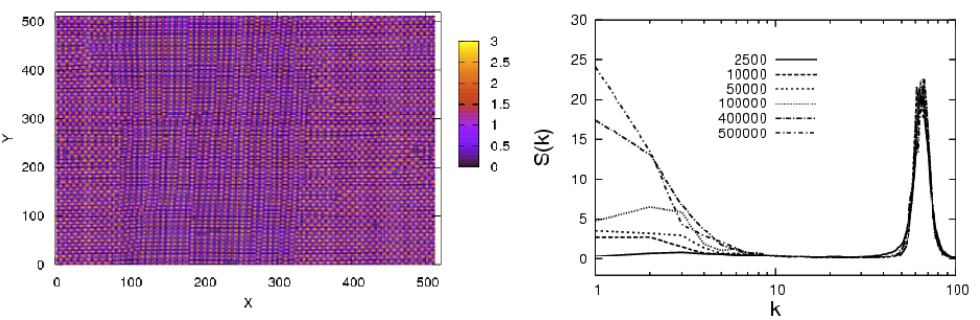

(c)

FIG. 3: Spatial distribution of the fluid density for the spray-emulsion configuration. As in previous picture, Fig. 2, $A_{1}=4.9$ in all cases and the corresponding Fourier spectra of density fluctuations are reported in the right panel. (a) $A_{2}=1.65, n x=n y=512, \mathrm{t}=500000$. (b) $A_{2}=2.15, n x=n y=512$, $\mathrm{t}=500$ 000. (c) $A_{2}=2.65, n x=n y=512, \mathrm{t}=500$ 000. Besides the sharp peak centered around the mean size of the droplets, the buildup of a low- $k$ component with increasing $A_{2}$ is well visible, corresponding to

the formation of large-scale domains indicating a higher degree of order in the global structure. The configuration presented in $(\mathbf{c})$ is strongly reminiscent of a crystal, with very few defects. For these cases, the typical radius is estimated as follows: (a) $R \approx 5.1$; (b) $R \approx 4.4$; (c) $R \approx 3.9$, [13].

that such spurious currents can be significantly tamed by the multi-range approach, the reason being that the repulsive forces only act on the first belt, and consequently they are only fourth-order isotropic. On the other hand, the Free-Energy approach, to be discussed below, seems better equipped to deal with this problem.

\section{THE FREE ENERGY APPROACH}

The Free Energy approach is mesoscopic in nature, as it derives from a density functional theory (a Cahn-Hilliard mixing energy density formulation for an isothermal system [[16]]). In its initial version, it was based on a heterogeneous local equilibrium including gradients of the fluid density such as to comply with the (continuum) free-energy functional for dense inhomogeneous fluids [2]. This approach is con- 


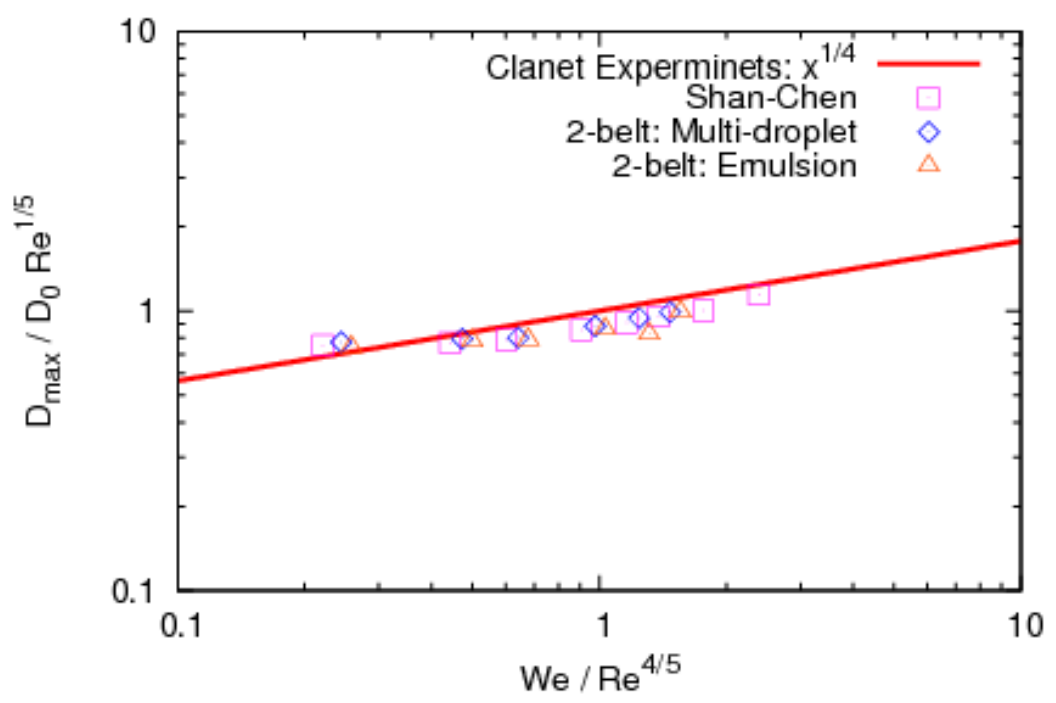

a)
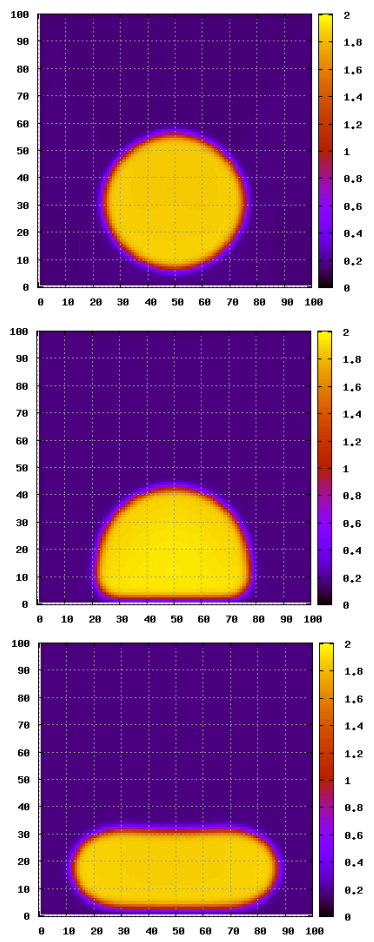

b)

FIG. 4: a) Comparison between the droplet deformations as a function of the Reynolds number Re, computed with the $L B$ method and the experimental results obtained by Clanet et al., [15] ; b) Sketch of drop impact on an hydrophobic wall, with $W e \sim 10$.

sidered as more fundamental than the PseudoPotential one, as it is derived from a Free-Energy functional. However, recent works [19] show that the Pseudo-Potential approach does indeed support a quasi-free energy functional and consequently, such distinction appears nowadays rather obsolete. More recently, the FE approach has been extended by Lee and coworkers, by making explicit use of an external force associated with non-ideal interactions, rather than the original heterogeneous local equilibrium $[20,21]$. The declared goal was to tame spurious currents, a severe limitations for many engineering applications. The basic quantities in Lee's approach are fluid pressure $P$ and composition $C$ (local mass fraction). In terms of the composition $C$ of one component, the free-energy functional reads as $E_{\text {mix }}(C, \nabla C)=E_{0}(C)+\frac{\kappa}{2}|\nabla C|^{2}$, where $\kappa$ is the gradient parameter.

The bulk energy can be rewritten as $E_{0}(C) \approx$ $\beta C^{2}(C-1)^{2}$ where $\beta$ is a constant fixing the free-energy barrier between the pure states $C=$ 0 and $C=1$. The same parameter fixes the non-ideal bulk pressure, through the Legendre's relation:

$$
p_{0}=C \frac{\partial E_{0}}{\partial C}-E_{0}
$$

The two free parameters $\beta$ and $\kappa$ provide separate control of the surface tension $\gamma$ and interface thickness $\delta$, respectively:

$$
\gamma=\frac{\sqrt{2 \kappa \beta}}{6}, \quad \delta=\sqrt{\frac{8 \kappa}{\beta}}
$$


The external force representing the non-ideal gas effects reads as follows:

$$
\mathbf{F}=\nabla \rho c_{s}^{2}-\nabla p_{0}+\rho \kappa \nabla \nabla^{2} \rho
$$

where the last term on the right hand side is directly responsible for surface tension effects.

The model by Lee evolves pressure instead of density, and consequently, the discrete distribution is defined as follows:

$$
g_{\alpha}=f_{\alpha} c_{s}^{2}+\left(p_{1}-\rho c_{s}^{2}\right) \Gamma_{\alpha}(0)
$$

where $f_{\alpha}$ is the usual discrete particle distribution, as defined in the classical LBE theory, and $\Gamma_{\alpha}(\mathbf{u})=f_{\alpha}^{e q} / \rho$, and $\alpha$ runs over the set of discrete speeds (in this application the twodimensional nine-speed lattice, D2Q9). In eq. (21) $p_{1}$ is the hydrodynamic pressure, which adds to the thermodynamic pressure $p_{0}$ and the curvature term to give the total pressure:

$$
P=p_{0}+p_{1}-\kappa C \nabla^{2} C+\frac{1}{2} \kappa|\nabla C|^{2}
$$

The distribution $g_{\alpha}$ is characterized by the following equilibrium function:

$$
g_{\alpha}^{e q}=w_{\alpha}\left[p_{1}+\frac{\mathbf{c}_{\alpha} \cdot \mathbf{u}}{c_{s}^{2}}+\frac{\left(\mathbf{c}_{\alpha} \cdot \mathbf{u}\right)^{2}}{2 c_{s}^{4}}-\frac{(\mathbf{u} \cdot \mathbf{u})}{2 c_{s}^{2}}\right]
$$

With a change of variables, the complete set of equations to be solved reads as follows:

$$
\begin{aligned}
& \bar{g}_{\alpha}=g_{\alpha}+\frac{1}{2 \tau}\left(g_{\alpha}-g_{\alpha}^{e q}\right) \\
& -\frac{\delta t}{2}\left(\mathbf{c}_{\alpha}-\mathbf{u}\right) \cdot\left[\nabla \rho c_{s}^{2}\left(\Gamma_{\alpha}-\Gamma_{\alpha}(0)\right)-C \nabla \mu \Gamma_{\alpha}\right]
\end{aligned}
$$

$$
\begin{gathered}
\bar{g}_{\alpha}^{e q}=g_{\alpha}^{e q} \\
-\frac{\delta t}{2}\left(\mathbf{c}_{\alpha}-\mathbf{u}\right) \cdot\left[\nabla \rho c_{s}^{2}\left(\Gamma_{\alpha}-\Gamma_{\alpha}(0)\right)-C \nabla \mu \Gamma_{\alpha}\right]
\end{gathered}
$$

Second-order integration in time (CrankNicolson), finally leads to the following LBE for pressure field:

$$
\begin{aligned}
\bar{g}_{\alpha}(\mathbf{x} & \left.+\mathbf{c}_{\alpha} \delta t, t+\delta t\right)-\bar{g}_{\alpha}(\mathbf{x}, t)= \\
& -\frac{1}{\tau+0.5}\left(\bar{g}_{\alpha}-\bar{g}_{\alpha}^{e q}\right)(\mathbf{x}, t) \\
& +\delta t\left(\mathbf{c}_{\alpha}-\mathbf{u}\right) \cdot \\
& \cdot\left[\nabla \rho c_{s}^{2}\left(\Gamma_{\alpha}-\Gamma_{\alpha}(0)\right)-C \nabla \mu \Gamma_{\alpha}\right]_{(\mathbf{x}, t)}
\end{aligned}
$$

The same procedure can be applied to the concentration $C$, by introducing a second distribution: $h_{\alpha}=\left(\frac{C}{\rho}\right) f_{\alpha}, h_{\alpha}^{e q}=\left(\frac{C}{\rho}\right) f_{\alpha}^{e q}$, which can be shown to obey the following LBE:

$$
\begin{aligned}
& \bar{h}_{\alpha}\left(\mathbf{x}+\mathbf{c}_{\alpha} \delta t, t+\delta t\right)-\bar{h}_{\alpha}(\mathbf{x}, t)= \\
& -\frac{1}{\tau+0.5}\left(\bar{h}_{\alpha}-\bar{h}_{\alpha}^{e q}\right)(\mathbf{x}, t) \\
& +\left.\delta t\left(\mathbf{c}_{\alpha}-\mathbf{u}\right) \cdot\left[\nabla C-\frac{C}{\rho c_{s}^{2}}\left(\nabla p_{1}+C \nabla \mu\right)\right] \Gamma_{\alpha}\right|_{(\mathbf{x}, t)} \\
& +\left.\delta t \nabla \cdot(M \nabla \mu) \Gamma_{\alpha}\right|_{(\mathbf{x}, t)}
\end{aligned}
$$

where the modified distribution $\bar{h}_{\alpha}$ and its equilibrium are calculated as in (24) and (25), namely:

$$
\begin{aligned}
\bar{h}_{\alpha}^{e q} & =h_{\alpha}^{e q} \\
& -\left.\frac{\delta t}{2}\left(\mathbf{c}_{\alpha}-\mathbf{u}\right) \cdot\left[\nabla C-\frac{C}{\rho c_{s}^{2}}\left(\nabla p_{1}+C \nabla \mu\right)\right] \Gamma_{\alpha}\right|_{(\mathbf{x}, t)} \\
& -\left.\frac{\delta t}{2} \nabla \cdot(M \nabla \mu) \Gamma_{\alpha}\right|_{(\mathbf{x}, t)}
\end{aligned}
$$

In the above $M$ is the mobility, a chemical factor in control of the rate of convergence to the equilibrium. The composition, the hydrodynamic pressure and the momentum are calculated by taking the zeroth and the first order moments of the modified particle distribution function:

$$
C=\sum_{\alpha} \bar{h}_{\alpha}+\frac{\delta t}{2} \nabla \cdot(M \nabla \mu)
$$




$$
\begin{gathered}
\rho c_{s}^{2} \mathbf{u}=\sum_{\alpha} \mathbf{c}_{\alpha} \bar{g}_{\alpha}-\frac{\delta t}{2} C \nabla \mu \\
p_{1}=\sum_{\alpha} \bar{g}_{\alpha}+\frac{\delta t}{2} \mathbf{u} \cdot \nabla \rho c_{s}^{2}
\end{gathered}
$$

The equation (29) is non linear, however, due to the slow variation of the chemical potential on the time-scale of a single time-step, in our implementation, $C$ at time $t$ is updated with the value of $\mu$ at the previous time-step $t-\delta t$, as suggested in [20]. The density and the relaxation time are calculated as local functions of the composition

$$
\begin{aligned}
& \rho(C)=C \rho_{1}+(1-C) \rho_{2} \\
& \tau(C)=C \tau_{1}+(1-C) \tau_{2}
\end{aligned}
$$

Technically, the distinctive feature of Lee's method, is the high-order finite-difference treatment of the non-ideal forces, at the righthand side of the evolution equations for the distribution functions. This is definitely more complicated and laborious than the Shan-Chen model, the reward being a drastic reduction of the spurious currents.

A. Free-Energy method: Numerical Simulations

Figure 5 shows the improvement obtained by the Free-Energy approach in terms of reducing the magnitude of the spurious currents. Notwithstanding the high density ratio (1:1000), the spurious currents remain of the order of $10^{-3}$. These results are definitely beyond reach of the SC approach, for which spurious currents are known to spoil the simulations already at density ratios around $50-100$.

Because of its superior stability, Lee's model is in a position to tackle more challenging multiphase problems than the SC model, at least

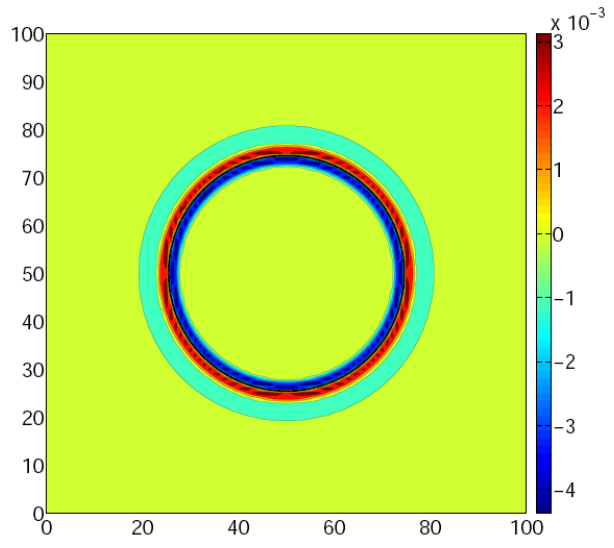

FIG. 5: Contour plot of the spurious force evaluated after $10^{6}$ time-steps. As expected, the spurious force is peaked around the interface. The main parameters are: radius $R=25$, liquid-vapor density ratio $\frac{\rho_{l}}{\rho_{v}}=1000$. The grid risolution is $100 \times 100,[23]$.

from an engineering point of view. As a very recent example, in Figure 6 we show the evolution of the fluid interface in a Rayleigh-Taylor instability at Reynolds number $R e=2048$, from He et al. [22] and from Lee model, respectively [23]. The flow field is qualitatively consistent with the typical RT instability dynamics, experimentally and numerically observed by various authors [24-26]: the initial exponential growth, the rise bubble of the light fluid and the spikes of denser fluid moving in the opposite direction as well as the superficial wave breaking at a later stage of the simulation, are well visible.

\section{CONCLUSIONS}

In this work, some basic features of two modern variants of the lattice Boltzmann method for non-ideal fluids have been discussed in connection with current and prospective micro-fluidic applications.

The Pseudo-Potential approach, with its most 


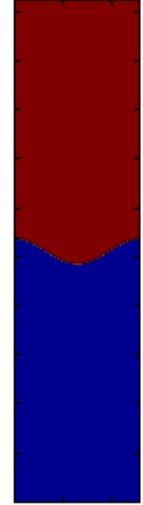

(a) $\mathrm{t}=0.0$

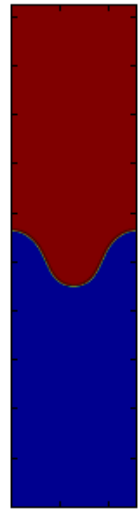

(b) $\mathrm{t}=1.0$

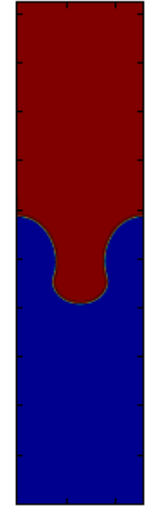

(c) $\mathrm{t}=1.5$

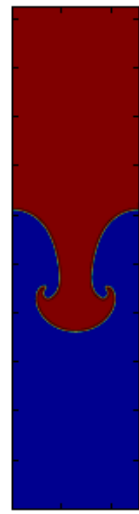

(d) $\mathrm{t}=2.0$
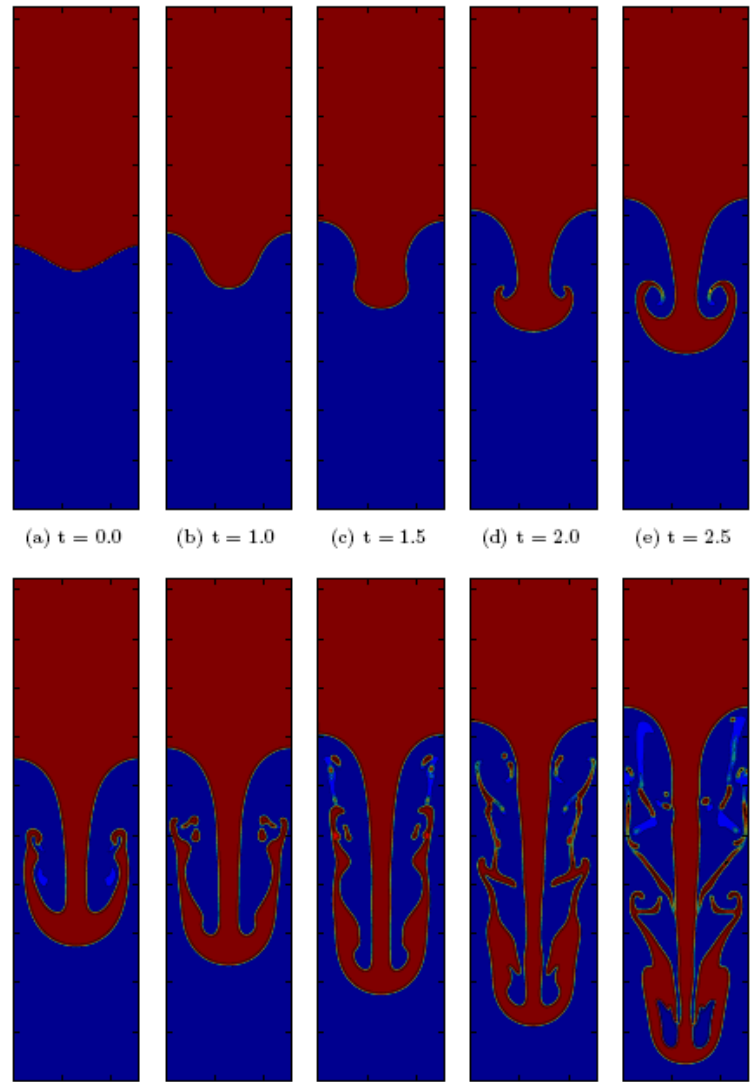

(f) $\mathrm{t}=3.0$

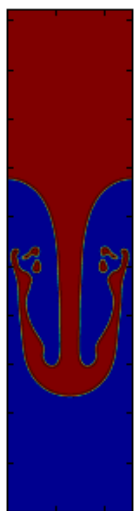

(g) $\mathrm{t}=3.5$

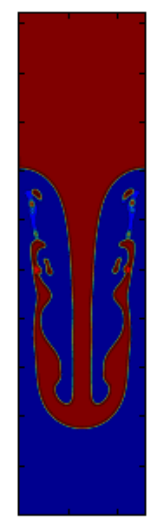

(h) $\mathrm{t}=4.0$

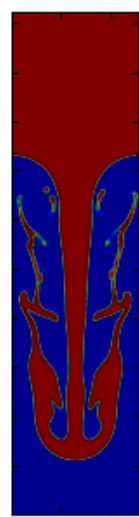

(i) $\mathrm{t}=4.5$

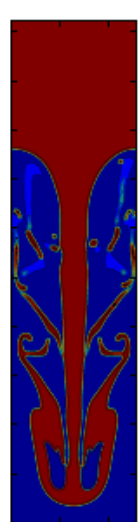

(j) $\mathrm{t}=5.0$

FIG. 6: Sequence of density contours for the RT instability at different times, as computed with Lee's model. The main parameters are: grid-size $256 \times 1024, R e=2048, A t=0.5$ and

$\sqrt{W \cdot g}=0.04$. Here $A t=\left(\rho_{l}-\rho_{v}\right) /\left(\rho_{l}+\rho_{v}\right)$ is the Atwood number, $W$ is the width of the domain and $g$ is the gravity field, [23].

recent multi-range enhancements, shows good

potential for the study of fundamental problems in micro-fluidics, involving complex flows with low interfacial tension, such as micro-emulsions and foams, as well as flows with complex fluidwall interactions, which bear special relevance to microfluidics.

The Free-Energy approach, in Lee's version, can handle a broader range of parameters, owing to its superior performance towards spurious currents and associated instabilities. Such superior performance must however be weighted against a corresponding increase of the computational burden, tipically a factor five in two dimensions. In addition, suitable boundary conditions in the presence of solid walls are still under development. Both examples, with their merits and limitations, witness the great vitality of the LB approach to the physics/engineering of multi-phase flows, at both macro and microscales.

\section{Acknowledgements}

S.S. wishes to acknowledge financial support from the European Project INFLUS, NMP3CT-2006-031980.
[1] Shan, X. \& Chen, H. Lattice Boltzmann model for simulating flows with multiple phases and components, Physical Review E, 47, 1993.

[2] Swift, M. R., Orlandini, E., Osborn, W. R. \& Yeomans, J. M. Lattice Boltzmann simulations of liquid-gas and binary fluid systems, Physical Review E, 54, 5041-5052 (1996).

[3] Lee, T. \& Fischer, P.F. Eliminating Parasitic currents in the lattice Boltzmann equation method for nonideal gasses, Physical Review E, 74046709 (2006).

[4] Benzi, R., Succi, S. \& Vergassola, M. The Lattice Boltzmann Equation: Theory and Applications, Phys. Rep., 222, 145-197 (1992).

[5] Chen, S. \& Doolen, G.D. Lattice Boltzmann method for fluid flows, Annu. Rev. Fluid Mech., 30, 329-364 (1998).

[6] Shan, X. Analysis of the spurious current near 
the interface in a class of multiphase lattice Boltzmann models, Physical Review E, 73, (2006).

[7] Qian, Y.H., D'Humieres, D. \& Lallemand, P. Lattice BGK Models for Navier-Stokes Equation, Europhys. Lett., 17(6):479-484, 1992.

[8] Succi, S. The lattice Boltzmann Equation for Fluid Dynamics and Beyond (Series Numerical Mathematics and Scientific Computation.Oxford University Press 2001).

[9] Shan, X. \& Chen, H. Simulation of nonideal gases and liquid-gas phase transitions by the lattice Boltzmann equation, Physical Review E, 49, 1994.

[10] Sbragaglia, M., Benzi, R., Biferale, L., Succi, S., Sugiyama, K. \& Toschi, F. Generalized lattice Boltzmann method with multirange pseudopotential, Physical Review E, 75, 026702 (2007).

[11] Falcucci, G., Bella, G., Chiatti, G., Chibbaro, S., Sbragaglia, M. \& Succi, S. Lattice Boltzmann Models with Mid-Range Interactions, Commun. Comput. Phys. 2, pp.1071-1084.

[12] Falcucci, G., Chiatti, G., Succi, S., Kuzmin, A. \& Mohamad, A.M. Rupture of a ferrofluid droplet in external magnetic fields using a single-component lattice Boltzmann model for nonideal fluids, Physical Review E, 79, 056706, (2009).

[13] Falcucci, G., Chibbaro, S., Succi, S., Shan, X. \& Chen, H. Lattice Boltzmann Spray-Like Fluids, Europhys. Lett., 82 (2008) 24005.

[14] Benzi, R., Biferale, L., Sbragaglia, M., Succi, S. \& Toschi, F. Mesoscopic modelling of heterogeneus boundary conditions for microchannel flows, J. Fluid Mech., 548, 257-280 (2006).

[15] Clanet, C., Bguin, C., Richard, D. \& Qur, D. Maximal deformation of an impacting drop, J. Fluid Mech., 517, 199-208, (2004).

[16] Jacqmin, D. Calculation of two-phase NavieStokes flows using phase-field modeling, J. Comp. Phys., 155, 96-127 (1999).

[17] Sbragaglia, M. \& Succi, S. Analytical calcula- tion of slip flow in lattice Boltzmann models with kinetic boundary conditions, Phys. Fluids, 17, 093602 (2005).

[18] Ansumali, S. \& Karlin, I.V. Kinetic boundary conditions in the lattice Boltzmann method, Physical Review E, 66, 026311 (2002).

[19] Sbragaglia, M., Chen, H., Shan, X. \& Succi, S. Continuum free-energy formulation for a class of lattice Boltzmann multiphase models, Europhys. Lett., 86, 24005 (2009)

[20] T.Lee, Effects of incompressibility on the elimination of parasitic currents in the lattice Boltzmann equation method for binary fluids, Computers and Mathematics with Applications (2009), doi:10.1016/j.camwa.2009.02.017.

[21] Lee, T. \& Liu, L. Wall boundary condition in the lattice Boltzmann equation method for nonideal gasses, Physical Review E, 78, 017702 (2008).

[22] He, X., Chen, S. \& Zhang, R. A Lattice Boltzmann Scheme for Incompressible Multiphase Flow and Its Application in Simulation of Rayleigh-Taylor Instability, J. Comp. Phys., 152, 642-663, (1999)

[23] Chiappini, D., Bella, G., Succi, S., Toschi, F. \& Ubertini, S. Improved Lattice Boltzmann without parasitic currents for Rayleigh-Taylor instability, Comm. in Comp. Phys. in press (2009).

[24] Nourgaliev, R.R., Dinh, T.N. \& Theofanous, T.G. A pseudo-compressibility method for the numerical simulation of incompressible multifluid flows, International Journal of Multiphase Flow, 30, (2004) 901-937.

[25] Cabot, W.H. \& Cook, A.W. Reynolds number effects on Rayleigh-Taylor instability with possible implications for type-Ia supernovae, Nature Physics Vol. 2 August 2006.

[26] Huang, Z., De Luca, A., Atherton, T.J., Bird, M. \& Rosenblatt, C. Rayleigh-Taylor Instability Experiments with Precise and Arbitrary Control of the Initial Interface Shape, Physical Review Letters, 99, 204502 (2007). 